\title{
A Computer Aided Case Tool for Temporal Knowledge Visualization
}

\author{
Yue Wang \\ University of Greenwich \\ London, United Kingdom \\ Y.wang@gre.ac.uk
}

\author{
Jixin Ma \\ University of Greenwich \\ London, United Kingdom \\ J.ma@gre.ac.uk
}

\begin{abstract}
The Internet has become a very important information source. Take Twitter for example: the tweets people post various kinds of data on the internet, usually with rich temporal and spatial aspects such as valid time, transaction time, location, and so on. In this paper we introduce a computer aided case tool alongside its theoretical underpinnings, for temporal knowledge visualization. It provides a user-friendly environment for describing scenarios with rich temporal information in natural language, which can be modeled in terms of Temporal Relationship Diagrams (TRD). The correspondence between sentences (and/or key phrases) in a given temporal scenario and the corresponding elements of the temporal relationship diagram can be automatically identified in a visualized way. From a temporal relationship diagram, the corresponding relative temporal relations and the equivalent "Meets" table can be derived automatically. A TRD can be automatically and visually transformed into its corresponding Time Graph, which is supported by an automatic consistency checker that delivers a visual and audio verdict as to whether a given scenario is temporally consistent or not.
\end{abstract}

Keywords- Artificial Intelligence; Temporal Representation; Knowledge Visuliazation

\section{INTRODUCTION}

The goal of artificial intelligence (AI) is to model human thinking and to embody those mechanisms in computers [1]. However, the ways to present human thinking have changed a lot in recent years. People, especially the young, are more likely to post their pictures and ideas on the social media. This means temporal logic should expand its application to such kinds of new field.

Fig.1 shows three important pieces of information as follows:

- This social media user likes Greenwich.

- The transaction time of this tweet is 10:04 AM on 13 January 2014.

- The location of the user sending this tweet is Greenwich, London.

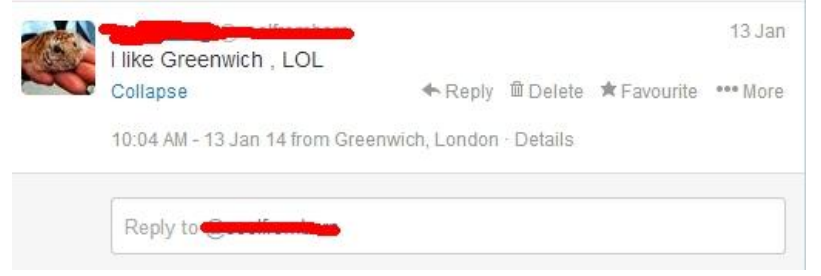

Figure 1. Social media example
Two questions naturally arise from Fig.1: (1) How to model the user's emotion? (2) How to visualize this information and reason about it?

The purpose of this paper is not to discuss question (1) (although there is no denying that it is crucially important) but instead focuses on question 2. The issue of how to combine the human-computer interaction (HCI) and $\mathrm{AI}$ in order to make it easy for non-experts to use is a big challenge. This paper argues that a user interface, which includes both a graphic interface designing for non-experts and a formulaic interface for experts, can intuitively help to solve this question.

In what follows in this paper, as the extension and implementation of the prototype work presented in [2], we introduce a computer aided case tool alongside its theoretical underpinnings. With this case tool, the user can model temporal scenarios either directly downloaded from social media such as Facebook, Twitter and so on, or presented in natural language in a visualized environment.

\section{THE TEMPORAL BASIS}

For general treatment, in this paper we shall adopt the time theory proposed in [3], which takes a nonempty set, Time, of primitive time elements, with an immediate predecessor relation, Meets, over time elements, and a duration assignment function, Dur, from time elements to non-negative real numbers. If $\operatorname{Dur}(\mathrm{t})=0$, then $\mathrm{t}$ is called a point; otherwise, if $\operatorname{Dur}(\mathrm{t})>0, \mathrm{t}$ is called an interval.

On one hand, such a point-and-interval based time theory overcomes problematic puzzles such as the so-called Dividing Instant Problem [4, 5, 6] due to treating intervals as derived structure from point based theories like that of Bruce [6]; on the other hand, it allows temporal references to both instantaneous phenomena with zero duration, and to periodic phenomena that last for some positive duration, and therefore no longer suffer from the limitations of Allen's interval based time theory [5], as pointed out by Galton [8].

Intuitively, the primitive relation Meets is simply an immediately predecessor relation over time elements. Therefore, Meets $\left(t_{1}, t_{2}\right)$ states that time element $t_{1}$ is one of the immediately predecessors of time element $t_{2}$, with no other time elements standing between them. Detailed axiomatization of this interval-and-point based time theory can be found in [3].

Analogous to the 13 relations introduced by Allen for intervals [5], there are 30 exclusive temporal order relations 
over time elements including both time points and time intervals:

- 3 relations relating a point to a point:

\{Equal, Before, After\}

- 7 relations relating a point to an interval:

\{Before, After, Meets, Met-by, Starts, During, Finishes

7 relations relating an interval to a point:

\{Before, After, Meets, Met-by, Started-y, Contains, Finished-by

- 13 relations relating an interval to an interval:

\{Equal, Before, After, Meets, Met-by, Overlaps,

Overlapped-by, Starts, Started-by, During, Contains,

Finishes, Finished-by\}

The definition of these derived temporal order relations in terms of the single relation Meets is straightforward. E.g.:

Before $\left(\mathrm{t}_{1}, \mathrm{t}_{2}\right) \Leftrightarrow \exists \mathrm{t} \in$ Time (Meets $\left.\left(\mathrm{t}_{1}, \mathrm{t}\right) \wedge \operatorname{Meets}\left(\mathrm{t}, \mathrm{t}_{2}\right)\right)$

\section{THE CASE TOOL}

Fig.2 shows the environment/use-interface of the case tool, called Visual Time, where the "File" button provides usual functions for file managements. Various views, including "Natural Language Description", "Time Relation Diagram (TRD)", "Temporal Relation", "Meets Table" and "Time Graph", are integrated in the framework, which can express given temporal scenarios in narrative form, diagrams and graphs.

In what follows, we demonstrate the main functions of the case tool by considering the following scenario:

Jack was suspected of committing a murder during the daytime. The policemen tried to collect evidence.

The policemen took the crime location as the centre to draw a circle on GPS map, and they found Jack was the only person who appeared within the circle. Fig.3 shows the interface of the GPS location.

By clicking the button "File" and choosing "import from social media", the user can download Jack's tweets from 12:00PM to 5:00PM of that day, and the result can be saved in Excel format, which is shown in Table I.

Fig.4 shows the interface of the downloading model interface, which is adopted from an open source blog [9] and will be only used for research purposes.

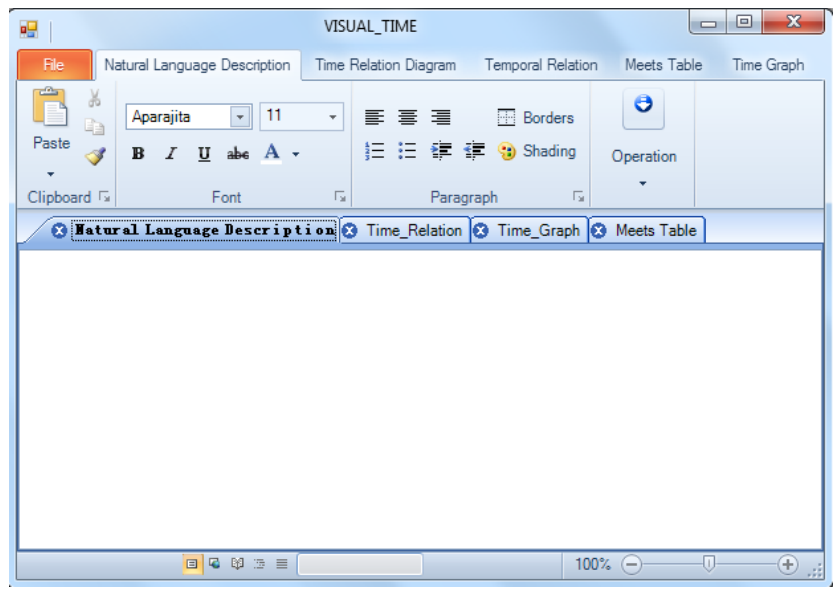

Figure 2. Visual Time environment

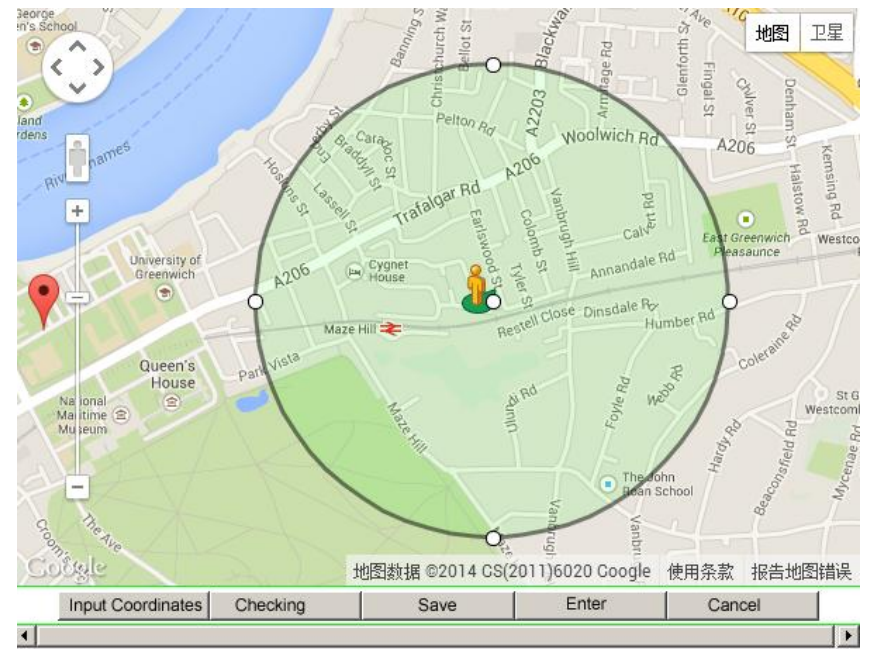

Figure 3. GPS loaction

TABLE I. JACK AND PETER'S TWEETS

\begin{tabular}{|c|c|c|c|}
\hline Date & Username & Tweets & Location \\
\hline $\begin{array}{c}\text { 2012-11-13 } \\
13: 00\end{array}$ & Jack & $\begin{array}{c}\text { Peter's home is } \\
\text { a standard geek } \\
\text { home, LOL }\end{array}$ & $\begin{array}{c}\text { SE100QQ(Peter's } \\
\text { home) }\end{array}$ \\
\hline $\begin{array}{c}\text { 2012-11-13 } \\
16: 00\end{array}$ & Jack & $\begin{array}{c}\text { Finally, Peter } \\
\text { arrived. }\end{array}$ & Greenwich Park \\
\hline
\end{tabular}

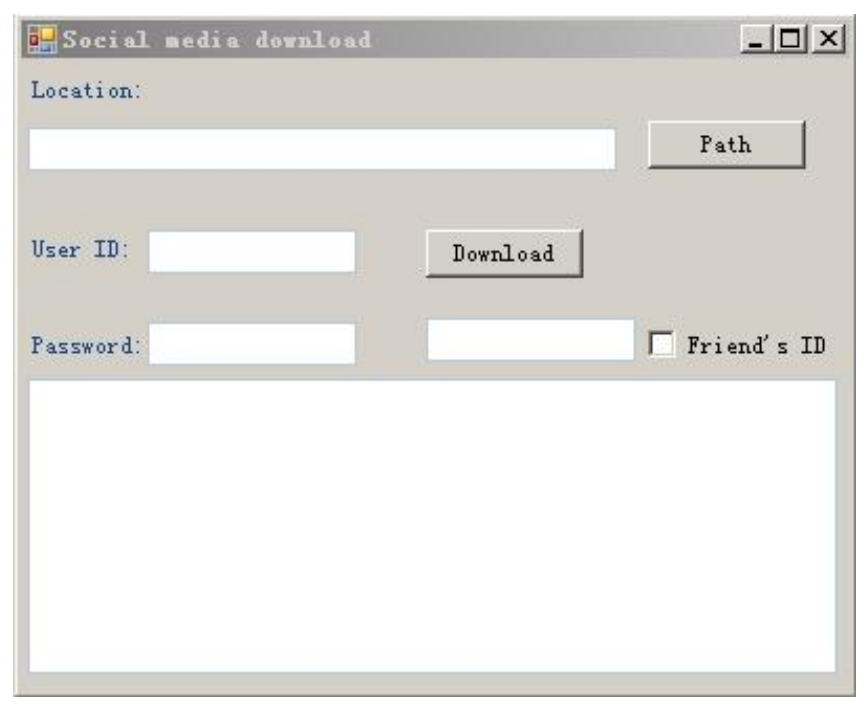

Figure 4. Social media download

Due to the fact that Jack's location was close to the crime location, the policemen decided to question him, and Jack gave the following statement in Fig.5.

Actually, in daily life, people rarely have and remember the exact (valid and/or transaction) time of every event. What they can tell are just some relative temporal information which is usually uncertain and incomplete. For 
example, a gentleman may just remember what he ate yesterday and roughly how long it took, but it is very hard (if not impossible) for him to tell the exactly starting time and end time of the "eating" activity. As for the statements illustrated in Fig.5, there is little knowledge about the exact times over which he conducted his activities.

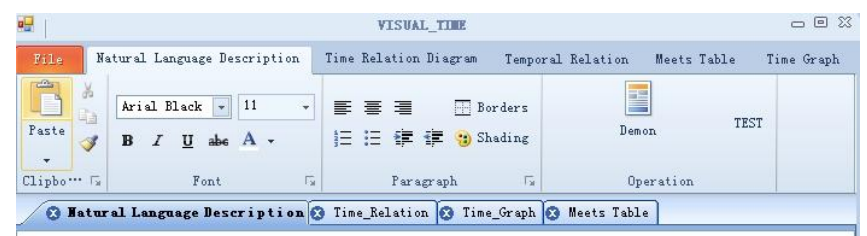

Peter and me went to his home. When we finished our lunch there, I left and went to the supermarket by bus or subway. I stayed there for about 1.5 hours. Then I drove to my home to collect some mail. It took about 2 hours to reach my home, and about the same to the Greenwich Park.

Figure 5. Natural Language Description

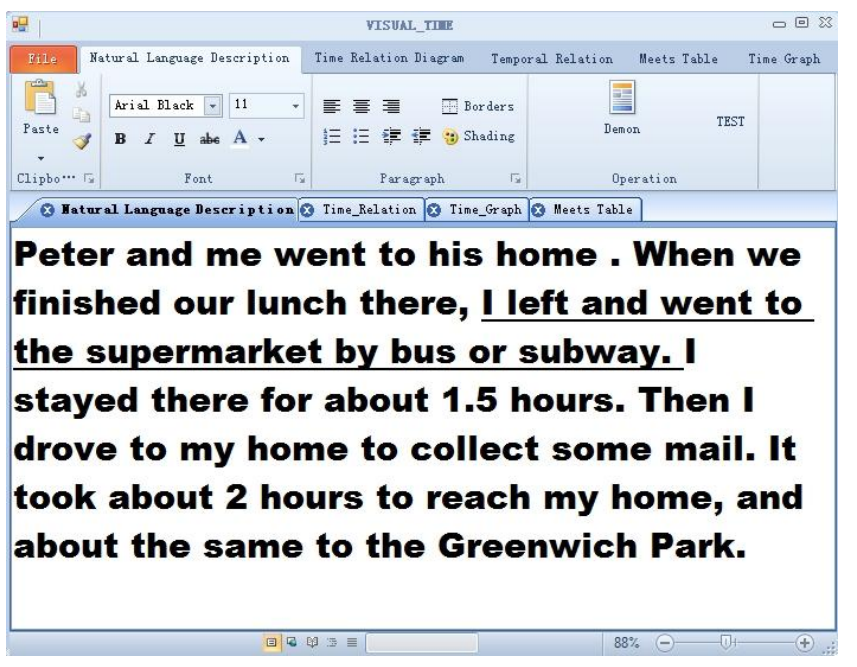

Figure 6. Underlined sentences
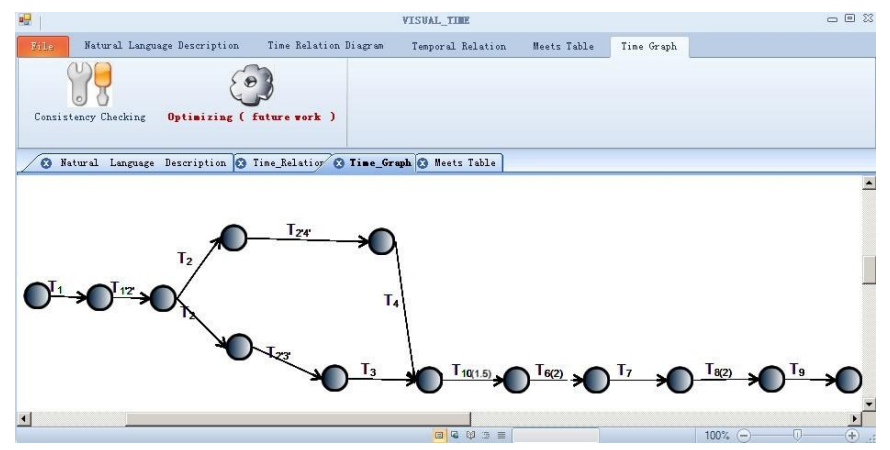

Figure 7. The graphical representation.
The Stanford parser is a very useful natural language processing (NLP) tool developed by the Stanford Natural Language Processing Group. It is an open source program which support Chinese, English and Arabic [10].

Using Stanford parser, it is not hard to pick out all the VBD or VBG from the scenario given in Fig.5.

Then I drove to my home to collect some mail. It took about 2 hours to reach my home

The above sentence shows that if we just use VBD and VBG to represent actions to draw the TRD, the sentence will be split into 4 actions - "drove", "collect", "took" and "reach". Because of this, a new algorithm was designed to solve this problem.

a) Use Stanford Temporal Tagger: SUTime to tag the natural language information.

b) Import the result into case tool.

c) Use a given dictionary to check every sentence, if it includes words like "COOL, FANTASTIC", delete those parts.

d) Check every sentence, if PRP of one sentence is "it" or "which", combine this sentence with its previous sentence.

e) Check every sentence, if it includes "here" or "there" with the Tag RB, find the first NN of previous sentence then change "here" or "there" with it.

f) Check every sentence, if VBD is followed by VBG, take VBG as the action. For example: "start working" or "start to work", "work" is the action, instead of "start".

g) Draw the TRD by every single VBD and VB.

h) Draw the TRD by the table got from social media.

The above algorithm can draw most of the time elements, except very complicated and long sentences. Because of the limitation of NLP, up to now, it has been impossible to obtain the relation between each time element. This paper can automatically draw most of the time elements from the social media (T2 and T9 in Fig.8) and natural language description (T1, T3, T4, T5, T6, T7, T8 and T10 in Fig.8), and the relations needs to be drawing manually.

Fig.8 shows the corresponding TRD of the above information.

The correspondence between sentences (and/or key phrases) in natural language description and the corresponding elements of TRD can be automatically identified in a visualized way. For example, if we click the button "Select Element", and choose time elements T3 and $\mathrm{T} 4$, the corresponding sentences will be underlined automatically in the natural language description as shown in Fig.6. On the other hand, if we choose some key phrases, sentences, or paragraphs in natural language description view, the corresponding time elements in TRD can be visually identified with bolded edges (as that of T3, T4 in Fig.8).

For a given TRD, its corresponding time graph [2] can be automatically drawn. Fig.7 shows the time graph of the TRD of Fig.8. In addition, the consistency checking function can deliver a visual and audio verdict as to whether a given scenario is temporally consistent or not [2]. 


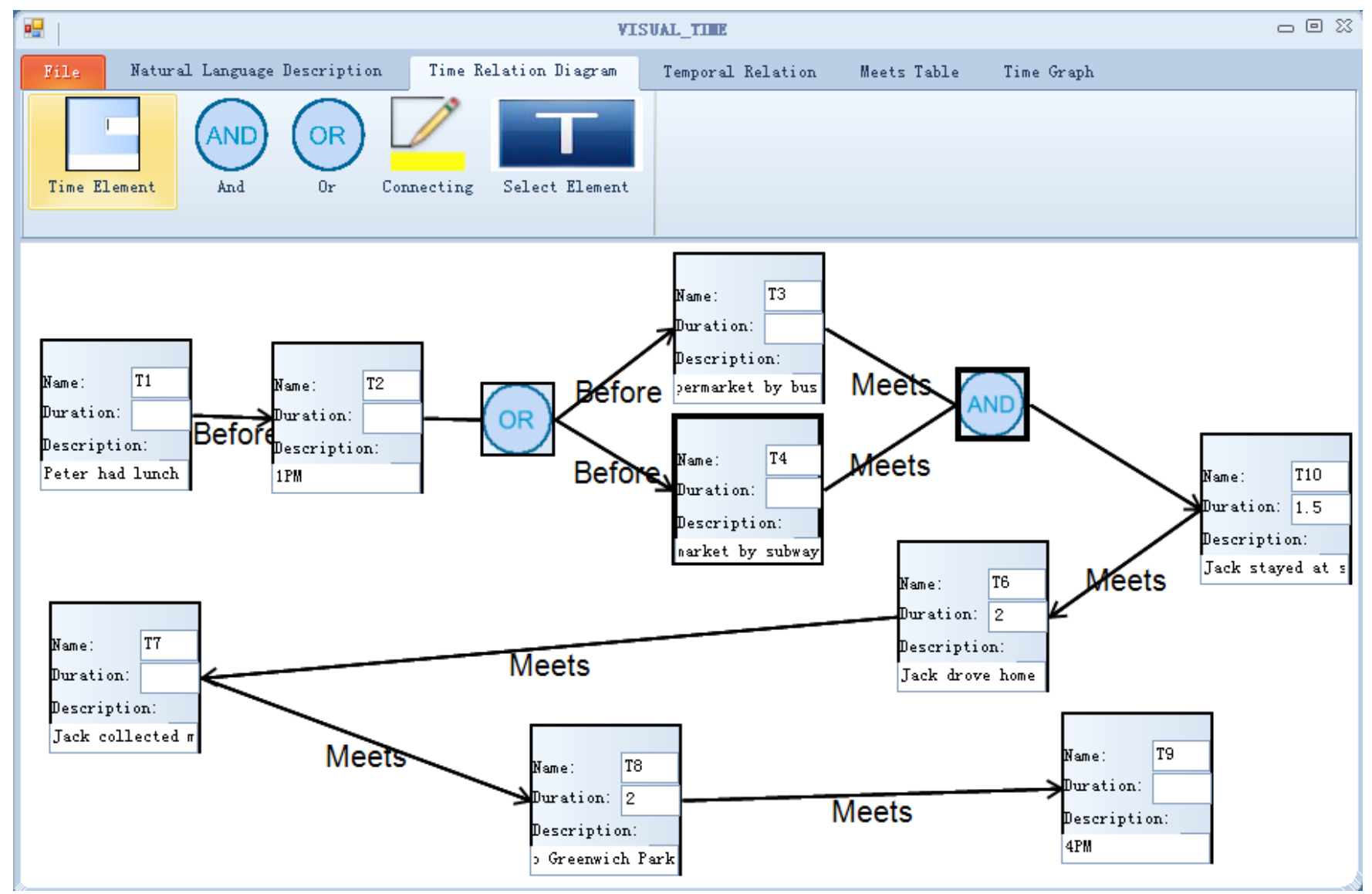

Figure 8. Time Relations Diagram

\section{CONCLUSION AND FUtURE WORK}

In this paper, we have presented a computer aided case tool for representing and reasoning about uncertain and incomplete temporal Knowledge. The user interface supports the user in modeling knowledge both from social media and given natural language descriptions. One can use it to graphically create and edit all of the time elements and their relations that can be stored and retrieved in TRD, which can be used as a logical base for many applications, e.g., Business Process Modeling, etc. This is an implementable system that is able to solve real-world (and full-scale) problems. It integrates interdisciplinary research activities including Temporal Logic, Natural Language Processing and Visual Modeling, etc.

\section{REFERENCE}

[1] Lieberman, Henry,User Interface Goals, $\mathrm{Al}$ Opportunities, AI Magazine, 30( 4), 2009, pp.16-22.

[2] Y. Wang, J. Ma and B. Knight: Implementing a Consistency Checker for Uncertain or Incomplete Temporal System, International Journal of Hybrid Information Technology, 6(6), 2013, pp.195-202.

[3] Jixin Ma and Brian Knight, A General Temporal Theory. the Computer Journal, 37(2),1994, pp.114-123.

[4] van J. Benthem, The Logic of Time, Kluwer Academic, Dordrech, 1983.

[5] James Allen. Towards a General Theory of Action and Time. Artificial Intelligence, 23, 1984, pp.123-154.

[6] J. Ma and B. Knight, Representing The Dividing Instant, the Computer Journal, 46(2), 2003, pp.213-222.

[7] B. Bruce. A model for temporal references and application in a question answering program. Artificial Intelligence, 3, 1972, pp.1-25.

[8] A. Galton, Critical Examination of Allen's Theory of Action and Time, Artificial Intelligence, 42, 1990, pp.159-188.

[9] http://www.blogjava.net/jayslong/archive/2010/11/17/sina_weibo_ba ckup.html

[10] http://nlp.stanford.edu/software/lex-parser.shtml 